\title{
99m Tc-Labeled Tricarbonyl His-CNA35 as an Imaging Agent for the Detection of Tumor Vasculature
}

\author{
Gilles Mees ${ }^{1}$, Rudi Dierckx ${ }^{1,2}$, Koen Mertens ${ }^{2}$, Simon Vermeire ${ }^{3}$, Magali Van Steenkiste ${ }^{4}$, Chris Reutelingsperger ${ }^{5}$, \\ Yves D’Asseler ${ }^{2}$, Kathelijne Peremans ${ }^{3}$, Nancy Van Damme ${ }^{6}$, and Christophe Van de Wiele ${ }^{1,2}$ \\ ${ }^{1}$ Department of Nuclear Medicine and Molecular Imaging, University Medical Center Groningen, University of Groningen, The \\ Netherlands; ${ }^{2}$ Department of Nuclear Medicine, Ghent University Hospital, Ghent, Belgium; ${ }^{3}$ Department of Medical Imaging, Ghent \\ University, Ghent, Belgium; ${ }^{4}$ Laboratory of Radiopharmacy, Ghent University, Ghent, Belgium; ${ }^{5}$ Department of Biochemistry, \\ Cardiovascular Research Institute, University of Maastricht, Maastricht, The Netherlands; and ${ }^{6}$ Department of Surgery, Ghent \\ University Hospital, Ghent, Belgium
}

\begin{abstract}
Given the importance of angiogenesis for a tumor's survival and growth, several therapeutic strategies rely on the selective inhibition of angiogenesis and the destruction of existing tumor vasculature. These strategies raise the need for a noninvasive tool to evaluate tumor vasculature. We describe the radiosynthesis and evaluation of an imaging tracer that specifically binds tumor subendothelial collagen and thereby images tumor vasculature. Methods: $99 \mathrm{mTc}$-tricarbonyl was prepared and labeled with His-collagen-binding adhesion protein 35 (CNA35). After in vitro specificity testing, in vivo biodistribution and dosimetric studies were performed in healthy nude mice via planar imaging. ${ }^{99 m} \mathrm{Tc}-(\mathrm{CO})_{3}$ His-CNA35 was evaluated for in vivo imaging of tumor vasculature in a HT29 colorectal carcinoma xenograft. Results: The labeling procedure yielded a compound with $95 \%-99 \%$ radiochemical purity and good in vitro stability. An in vitro binding test confirmed specificity and functionality. $99 \mathrm{mTc}-(\mathrm{CO})_{3}$ His-CNA35 rapidly cleared from the blood and predominantly accumulated in the kidneys and liver. The effective dose for a proposed single injection of $500 \mathrm{MBq}$ of ${ }^{99 \mathrm{mTc}-(\mathrm{CO})_{3}}$ His-CNA35 is $3.70 \mathrm{mSv}$ per organ or $2.01 \mathrm{mSv} / \mathrm{g}$ of tissue. Tumors were successfully visualized, and uptake correlated with ex vivo immunohistochemical staining of tumor vasculature.

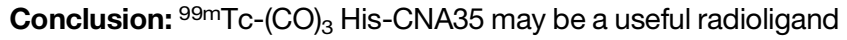
for the in vivo detection of tumor vasculature through subendothelial collagen binding. A noninvasive method of imaging tumor vasculature that could provide a reliable assessment of tumor vasculature would allow evaluation of the effectiveness of commonly used antiangiogenic therapies and determination of their optimal dosing and scheduling.
\end{abstract}

Key Words: CNA35; tricarbonyl; tumor vasculature; HT29

J Nucl Med 2012; 53:464-471

DOI: 10.2967/jnumed.111.095794

\footnotetext{
Received Jul. 18, 2011; revision accepted Nov. 1, 2011.

For correspondence or reprints contact: Christophe Van de Wiele, Department of Nuclear Medicine, Ghent University Hospital, De Pintelaan 185, 9000 Ghent, Belgium.

E-mail: christophe.vandewiele@ugent.be

Published online Feb. 13, 2012.

COPYRIGHT @ 2012 by the Society of Nuclear Medicine, Inc.
}

A a tumor mass grows beyond the support capacity of the vasculature it relies on, the need for new blood vessels arises. The formation of new blood vessels is achieved through angiogenesis, a multistep process that relies on the tumor-driven production of proangiogenic factors. These resulting new blood vessels, however, are structurally and functionally deficient when compared with normal ones. They are disorganized, tortuous, and leaky, leading to heterogeneous blood flow, hypoxia, acidosis, and elevated interstitial fluid pressure, with a disturbed tumor microenvironment as a result (1). Nevertheless, this neovascularization allows the tumor to grow and plays an important role in tumor invasiveness and metastasis. Given the importance of angiogenesis, several therapeutic strategies rely on the selective inhibition of angiogenesis and destruction of tumor vasculature. In several types of cancer, antiangiogenic therapy alone or in combination with standard chemotherapeutic strategies has led to an improvement of survival $(2,3)$. As the importance of these therapies increases, noninvasive methods are needed to provide a reliable assessment of tumor vasculature and thus a means for the management and planning of antiangiogenic therapy.

A possible mechanism to target tumor vasculature is based on the selective exposure of collagen in these vessels due to their aberrant endothelial lining. Collagen-binding adhesion protein 35 (CNA35) is a 35-kDa collagen-binding domain of specific bacterial adhesion proteins from Staphylococcus aureus and participates in the infectious process of this bacteria. The collagen-binding properties of these domains are well characterized, and overexpression can be attained in Escherichia coli in large quantities (4,5). CNA35 has previously been labeled with several fluorophores to visualize collagen in tissues and life cell cultures and has a moderate affinity $(\sim 0.5 \mu \mathrm{M})$ for a variety of different collagen types (5-7). The proposed mechanism of binding involves the wrapping of the CNA35 protein around the triple helix of collagen (4). Collagens are an important component of the extracellular matrix, an interconnected molecular network providing mechanical support for cells and 
tissues and regulating certain biochemical and cellular processes. Until now, 19 different collagens-categorized as fibrillar or nonfibrillar-have been identified. Collagen IV is the major component of the vascular basement membrane, a specialized form of extracellular matrix that lines the blood vessels and separates epithelia from its underlying mesenchyme (8). In contrast to normal blood vessels, tumor vasculature displays an abnormal, incomplete endothelial lining that is characterized by large interendothelial junctions and an increased number of fenestrations (1), allowing CNA35 to penetrate the endothelial lining and bind the underlying collagen layer. In normal vessels, these abnormalities do not exist to this degree, preventing major infiltration of the endothelial lining and subsequent binding of the underlying collagen layer. As a result, specific targeting of tumor vasculature is possible, and noninvasive imaging of tumor vasculature is feasible when labeling CNA35 with a radioactive molecule.

The aim of this study was to radioactively label CNA35 and evaluate the use of this marker in the selective, noninvasive imaging of tumor vasculature.

\section{MATERIALS AND METHODS}

\section{${ }^{99 m} \mathrm{Tc}-(\mathrm{CO})_{3}$ His-CNA35 Preparation}

The labeling precursor ${ }^{99 m}$ Tc-tricarbonyl $\left(\left[{ }^{99 m} \mathrm{Tc}(\mathrm{CO})_{3}\left(\mathrm{OH}_{2}\right)\right.\right.$ $3]^{+}$) was prepared according to the manufacturer's instructions. One milliliter of eluted ${ }^{99} \mathrm{TcO}_{4}{ }^{-}(1,480-2,590 \mathrm{MBq})(\mathrm{GE}$ Healthcare) was added to the IsoLink vial (IsoLink kits were kindly provided by Mallinckrodt Medical B.V.) containing the following lyophilized products: $4.5 \mathrm{mg}$ of sodium boranocarbonate, $2.85 \mathrm{mg}$ of sodium tetraborate $10 \mathrm{H}_{2} \mathrm{O}$, and $8.5 \mathrm{mg}$ of sodium tartrate $\cdot 2 \mathrm{H}_{2} \mathrm{O}$. The vial was placed in a water bath for $30 \mathrm{~min}$ at $95^{\circ} \mathrm{C}-100^{\circ} \mathrm{C}$, after which the reaction vial was cooled to room temperature. The precursor was brought to $\mathrm{pH} 7$ by the addition of $1 \mathrm{M} \mathrm{HCl}$. His-CNA35 (50-60 $\mu \mathrm{g}$ ) (provided by Prof. Dr. Chris Reutelingsperger, Department of Biochemistry, University Maastricht) was added to the precursor and incubated at $37^{\circ} \mathrm{C}$ for 1-1.5 h while blown dry with nitrogen $\left(\mathrm{N}_{2}\right)$ gas to reduce the reaction volume. Protein LoBind Tubes (Eppendorf) were used to prevent adhesion of the protein to the vial wall. The vial was washed with physiologic saline $(0.9 \% \mathrm{NaCl})$, and the reaction mixture was purified on a Sephadex G-25 medium PD-10 column (GE Healthcare Bio-Sciences AB), which was activated with phosphate-buffered saline (PBS) containing $0.5 \%$ bovine serum albumin (BSA) (Sigma-Aldrich-Fluka) and eluted with fractions of $500 \mu \mathrm{L}$ of PBS. The radioactivity of each fraction was measured in a dose calibrator (VIK-202; Veenstra).

\section{Radiochemical Analysis}

The radiochemical purity of the ${ }^{99 \mathrm{~m}} \mathrm{Tc}-(\mathrm{CO})_{3}$ His-CNA35 was determined by instant thin-layer chromatography (ITLC) with silica gel-coated fiberglass sheets (Life Sciences, Pall Corp.) using physiologic saline as the mobile phase. Samples of radiolabeled His-CNA35 were tested for their in vitro stability at 4 and $24 \mathrm{~h}$ after preparation at $4^{\circ} \mathrm{C}$ and $37^{\circ} \mathrm{C}$ in PBS, McCoy $5 \mathrm{~A}$ cell medium supplemented with $10 \%$ fetal bovine serum, and human blood serum. Fifty microliters of ${ }^{99 \mathrm{~m}} \mathrm{Tc}-(\mathrm{CO})_{3}$ His-CNA35 was mixed with $50 \mu \mathrm{L}$ of PBS, cell medium, or serum, and ITLC was performed to assess the radiochemical purity. At both times, $5 \mu \mathrm{L}$ of the samples were spotted on ITLC strips, and radioactivity was counted using an automated $\gamma$-counter (Cobra II series; Canberra Packard). High-pressure liquid chromatography (HPLC) was performed to analyze the integrity and radiochemical purity of the radiolabeled CNA35, with a size-exclusion Shodex KW 802.5 column $(7.8 \times 300 \mathrm{~mm}$; Thomsom Instrument Co. $)$ connected to an ultraviolet-visible spectrometer $(\lambda=280 \mathrm{~nm})$ (SPD-6AV; Shimadzu) and a NaI $\gamma$-counter (model 2200; Ludlum). Elution was performed at a flow rate of $0.8 \mathrm{~mL} / \mathrm{min}$ with a $0.1 \mathrm{M}$ potassium phosphate buffer, $\mathrm{pH} 7$, and a 510 dual-head pump (Waters). The purity of the unlabeled and labeled compound was further analyzed with sodium dodecyl sulfate polyacrylamide gel electrophoresis (SDS-PAGE), performed according to the method of Laemmli (9). The labeled (0.04 MBq) ${ }^{99 m} \mathrm{Tc}-(\mathrm{CO})_{3}$ His-CNA35 and unlabeled $(5 \mu \mathrm{g})$ His-CNA35 were mixed with an equal volume of Laemmli sample buffer $(2 \times)$ and loaded onto a $15 \%$ polyacrylamide gel. Ten microliters of a protein ladder (10-250 kDa) from Biolabs was treated the same way. Electrophoresis was performed at $200 \mathrm{~V}$ (Protean III system; Bio-Rad) until the dye front reached the bottom of the gel. The separated proteins were visualized either with Coomassie Brilliant Blue R-250 staining (Bio-rad) or by exposure of a superresolution film for $1 \mathrm{~min}$ to the radioactive gel, after which visualization of the radioactive protein was effected with Cyclone Storage Phosphor equipment (PerkinElmer).

\section{In Vitro Solid-Phase Binding Assay}

Collagen I-, collagen IV-, laminin-, and fibronectin-coated multiwell plates (BD Biosciences) were blocked with 3\% PBS/ BSA for $3 \mathrm{~h}$ at $37^{\circ} \mathrm{C}$ and aspirated and washed 3 times with PBS. Afterward, increasing concentrations of ${ }^{99 \mathrm{~m}} \mathrm{Tc}-(\mathrm{CO})_{3}$ His-CNA35 were added to each well and then incubated for $3 \mathrm{~h}$ at $37^{\circ} \mathrm{C}$. Supernatant was removed, and wells were washed 2 times with PBS. Supernatant was measured using an automated $\gamma$-counter (Cobra II series; Canberra Packard), and uptake was calculated. To assess nonspecific binding, uptake was measured in a noncoated multiwell plate (BD Biosciences) as well. All assays were performed in triplicate.

\section{HT29 Cell Culture}

HT29 human colorectal carcinoma cells (ACC 299; DSMZ) were cultured in McCoy 5A medium (Gibco) supplemented with $10 \%$ fetal bovine serum (Invitrogen Corp.), $4 \mathrm{mM} \mathrm{L-glutamine,}$ penicillin $(50 \mu \mathrm{L} / \mathrm{mL})$, and streptomycin $(50 \mu \mathrm{g} / \mathrm{mL})(\mathrm{Gibco})$ and incubated at $37^{\circ} \mathrm{C}$ in a humidified atmosphere of $5 \% \mathrm{CO}_{2}$ in air. Cells were kept in exponential growth phase by routine passage every 3-4 d (split ratio, 1/4-1/6).

\section{Animal Model}

Female CD-1 nude mice (4-6 wk old) were purchased from Charles River Laboratories and housed in a standard facility at the Department of Animal Sciences, Ghent University Hospital. All procedures were performed in accordance with the guidelines and regulations for use and care of animals and approved by the local ethical committee, Faculty of Medicine, Ghent University (EC 09/ 14). One million HT29 human colorectal carcinoma cells suspended in $0.1 \mathrm{~mL}$ of PBS were injected subcutaneously in the proximal right hind leg. Tumor growth curves were obtained using caliper measurement and the estimate volume formula $\left(\mathrm{mm}^{3}\right)=$ $1 / 2\left(a^{2} b\right)$, where $a$ and $b$ were the short and long axis of the tumor, respectively. Experiments were initiated when tumors reached approximately $10 \mathrm{~mm}$ in diameter, generally $14 \mathrm{~d}$ after implantation. 


\section{Biodistribution and Dosimetry of Radiolabeled CNA35}

For biodistribution and dosimetry studies, 4 healthy nude mice were positioned on the $\gamma$-camera, and isoflurane anesthesia (2\%) and body temperature were maintained for the duration of the experimental procedure. After bolus tail-vein administration of $37 \mathrm{MBq}(\sim 4 \mu \mathrm{g})$ of ${ }^{99 \mathrm{~m}} \mathrm{Tc}-(\mathrm{CO})_{3}$ His-CNA35 in $150 \mu \mathrm{L}$, mice were imaged during the first 100 min (dynamic study, 100 frames of $1 \mathrm{~min}$ ), followed by planar images of $10 \mathrm{~min}$ at 4,8 , and $23 \mathrm{~h}$ after injection of the radiotracer for evaluation of the biodistribution of ${ }^{99 \mathrm{~m} T c}-(\mathrm{CO})_{3}$ His-CNA35 in vivo. Mice were imaged using a conventional triple-head $\gamma$-camera (Triad; Trionix) operated in planar mode with the animals positioned on 1 of the 3 detectors. Regions of interest (ROIs) were drawn over the total body, liver, right kidney, left kidney, heart, and part of the left hind leg (background) using PMOD software (version 3.0; PMOD Technologies). ROIs were drawn on early images, and the shape and size (number of pixels) were kept constant over all subsequent images per mouse. Total body retention was estimated using the no-excretion approach - that is, total counts collected during the first minute of the dynamic study were assumed to correspond to the total injected dose. Total counts from a later whole-body scan at time $t_{i}$ were used to estimate percentage injected dose (\%ID): (counts $t_{i} /$ counts [first frame] $\times 100 \%)$. After correction for background radiation, decay, and, when needed, residual activity in the tail, uptake in the organs was expressed as \%ID.

On the basis of quantification of whole-body images (0$100 \mathrm{~min}, 4 \mathrm{~h}, 8 \mathrm{~h}$, and $23 \mathrm{~h}$ ) from the biodistribution studies, dosimetry of ${ }^{99 \mathrm{~m}} \mathrm{Tc}-(\mathrm{CO})_{3}$ His-CNA35 was estimated using MIRDose 3.1. Organ residence time was obtained by integrating the organ activity curve that was normalized with the injected activity but not corrected for physical decay. For liver, right kidney, left kidney, and the remainder of the body, time-activity curves were constructed from the dynamic (first $100 \mathrm{~min}$ ) and static planar scans at later times. The time-activity curves were fitted to biexponential functions, and residence times were calculated. On the basis of these, organ dose and effective dose (ED) were calculated with MIRDose 3.1. Data obtained from mice were converted to a 68-kg human reference adult, both using an activity-per-organ and an activity-per-mass approach.

\section{In Vivo Imaging of Tumor Vasculature in Human HT29 Colorectal Tumor Xenograft Model}

For in vivo imaging of tumor vasculature, 10 HT29 colorectal carcinoma xenografts were injected intravenously with $18.5 \mathrm{MBq}$ $(\sim 2 \mu \mathrm{g})$ of ${ }^{99 \mathrm{mTc}}-(\mathrm{CO})_{3}$ His-CNA35 in $150 \mu \mathrm{L}$. Scanning began $3 \mathrm{~h}$ after injection, with isoflurane anesthesia (2\%) and body temperature maintained throughout the scan. A 20-min planar static scan was obtained using a conventional triple-head $\gamma$-camera (Triad; Trionix) with the animals positioned on 1 of the 3 detectors. A syringe with a known amount of radioactivity was scanned along with the mice to allow semiquantification of the results using ROI analysis. ROIs were copied from the tumor-containing right hind leg to the left hind leg, and data were expressed as tumor-tobackground ratio, using the left hind leg as background. Directly after the scan, the animals were killed by cervical dislocation, and tumors were removed, formalin-fixed, and paraffin-embedded.

\section{Detection of Tumor Vasculature by Immunohistochemistry}

Tumor vasculature was evaluated using immunohistochemical markers for endothelial cells (CD105/Endoglin; AF1320 [R\&D Systems], 1/50), blood vessel basement membrane (collagen IV; ab6586 [Abcam], 1/500), and mural cells-that is, pericytes and vascular smooth muscle cells ( $\alpha$-smooth muscle actin [ $\alpha$-SMA], ab5694 [Abcam], 1/200). Tissue sections of $5 \mu \mathrm{m}$ thick were mounted on SuperFrost microscope slides (Menzel-Glaser), which were deparaffinized in xylene and rehydrated in a downgraded series of ethanol. After the slides were washed in the appropriate buffer (tris-buffered saline with $0.1 \%$ polysorbate 20 ), heat-induced antigen retrieval was performed for $20 \mathrm{~min}$ in citrate buffer ( $\mathrm{pH}$ 6.0), after which the slides were cooled for $15 \mathrm{~min}$. The endogenous peroxidase activity was blocked for $5 \mathrm{~min}$ with $0.3 \%$ hydrogen peroxide (Dako). Slides were then incubated with the primary antibody overnight (CD105) or for $1 \mathrm{~h}$ (collagen IV and $\alpha$-SMA) at room temperature (primary antibody diluted in PBS with 1\% BSA). After being washed, the tissue sections were incubated for $30 \mathrm{~min}$ at room temperature with a horseradish peroxidase-labeled antirabbit secondary antibody (Dako) or the combination of a biotinylated link and streptavidin-horseradish peroxidase (Dako). The color reaction was developed using the chromogen 3,3-diaminobenzidine (Dako) for $30 \mathrm{~s}$. After being washed, the tissue sections were counterstained with Mayer hematoxylin. To exclude false-positive responses from nonspecific binding of the secondary antibody, negative controls were tested with $1 \%$ PBS-BSA instead of the primary antibody. Before the specimens were stained, an isotype control was performed to estimate the nonspecific binding of target primary antibodies to cellsurface antigens.

\section{Immunohistochemical Analysis}

An Optronicscolor digital camera (Olympus Corp.) and specialized software (Cell D Olympus Imaging Solutions) were used to analyze immunohistochemical stainings. Microvessel density (MVD) was assessed in sections stained for CD105 using the criteria of Weidner et al. (10). Briefly, the entire tumor section was scanned at low power (magnification, $\times 40$ ) to identify vascular hot spots, which are the areas of highest vascularization. Individual microvessels were then counted under high power (magnification, $\times 200$ ) to obtain a vessel count in a defined area, and the average vessel count in 5 hot spots was taken as the MVD. Any brownstained endothelial cell or endothelial cell cluster that was clearly separated from adjacent microvessels, tumor cells, and other connective tissue elements was considered a single, countable microvessel. The same hot spots were used to count $\alpha$-SMA and collagen IV-positive vessels using the same procedure. A ratio of $\alpha$-SMA to CD105 was used to calculate the vessel maturity index (VMI), which provided a percentage of mature vessels (11).

\section{Statistical Analysis}

All statistical analysis was performed using SPSS software (version 15.0; SPSS Inc.). A Spearman correlation test was used to calculate correlations between ${ }^{99 \mathrm{~m}} \mathrm{Tc}-(\mathrm{CO})_{3}$ His-CNA35 tumor uptake and immunohistochemical stainings in the tumor. Data were considered statistically significant when a 2 -tailed $P$ value of less than 0.05 was reached.

\section{RESULTS}

\section{Radiochemical Profile of ${ }^{99 m} \mathrm{Tc}-(\mathrm{CO})_{3}$-His CNA35}

His-tagged CNA35, with an N-terminal extension containing 6 histidine residues, was successfully labeled with ${ }^{99 \mathrm{~m}} \mathrm{Tc}-(\mathrm{CO})_{3}$. Incubation of the His-CNA35 with the ${ }^{99 \mathrm{~m}} \mathrm{Tc}-$ $(\mathrm{CO})_{3}$ occurred at physiologic $\mathrm{pH}(7)$ and at a temperature 
of $37^{\circ} \mathrm{C}$ for about $1-1.5 \mathrm{~h}$. Although specialized vials were used to prevent adhesion of the protein to the vial wall, considerable residual activity was noted in the reaction vial. Radiochemical yields of $40 \%-50 \%$ and specific radioactivities of approximately 8.9-10.4 MBq of protein per microgram were obtained. ${ }^{99 \mathrm{~m}} \mathrm{Tc}-(\mathrm{CO})_{3}$ His-CNA35 was purified on a calibrated PD-10 gel permeation column using PBS as eluent. The radiochemical purity of the labeled CNA35 was evaluated by ITLC, with physiologic saline as mobile phase. Radiochemical purities of $95 \%-99 \%$ were obtained, with the remainder being free ${ }^{99 \mathrm{~m}} \mathrm{Tc}$ pertechnetate $(1 \%-$ 4\%). HPLC was performed to characterize the radiolabeled

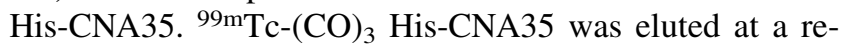
tention time of $9.8 \mathrm{~min}$. SDS-PAGE showed a band of 36 $\mathrm{kDa}$ representing the radiolabeled His-CNA35. No other small molecules were identified. In vitro, no significant degradation of the product was noted for up to $24 \mathrm{~h}$, at $4^{\circ} \mathrm{C}$ and $37^{\circ} \mathrm{C}$, in PBS, cell medium supplemented with $10 \%$ fetal bovine serum, or human blood serum. The stability tests thus demonstrated that ${ }^{99 \mathrm{~m}} \mathrm{Tc}-(\mathrm{CO})_{3}$ His-CNA35 has good thermodynamic stability in PBS, cell medium, and human blood serum.

\section{In Vitro Solid-Phase Binding Assay}

Binding of ${ }^{99 \mathrm{~m}} \mathrm{Tc}-(\mathrm{CO})_{3}$ His-CNA35 to collagen I-, collagen IV-, laminin-, and fibronectin-coated multiwell plates was studied to confirm the specificity and functionality of the labeled molecule. Results are summarized in Figure 1. Binding of ${ }^{99 \mathrm{~m}} \mathrm{Tc}-(\mathrm{CO})_{3}$ His-CNA35 to collagen I and IV was observed. Negligible binding was observed to laminincoated, fibronectin-coated, and noncoated surfaces, possibly the result of nonspecific adhesion to the plastic of the multiwall plates.

\section{Biodistribution}

After bolus tail-vein administration of $37 \mathrm{MBq}$ of ${ }^{99 \mathrm{~m} T c-}$ $(\mathrm{CO})_{3}$ His-CNA35, mice were imaged during the first $100 \mathrm{~min}$ (dynamic study, 100 frames of $1 \mathrm{~min}$ ), followed by planar images of $10 \mathrm{~min}$ at 4,8 , and $23 \mathrm{~h}$ after injection of the radiotracer. The biodistribution of ${ }^{99 \mathrm{~m}} \mathrm{Tc}-(\mathrm{CO})_{3}$ HisCNA35 in 4 healthy nude mice is summarized in Table 1 and Figure 2. After intravenous injection of ${ }^{99 \mathrm{~m}} \mathrm{Tc}-(\mathrm{CO})_{3}$ His-CNA35, the radiotracer was rapidly cleared from the blood $(6.54 \pm 1.47 \%$ ID during the first minute, with only $1.86 \pm 0.14 \%$ ID remaining in the blood at $1 \mathrm{~h}$ after injection). Liver and kidneys showed the highest radiotracer uptake throughout the biodistribution experiment (Table 1; Figs. 2 and 3). At all times, the right kidney showed a higher uptake than the left kidney. Thus, renal excretion appears to be the main route of radiotracer clearance.

\section{Dosimetry}

To translate the mouse biodistribution data into estimates of patient dose, the organ values were extrapolated from the data derived from the biodistribution data to a $68-\mathrm{kg}$ man using the standard MIRD scheme and S tables. A summary of dosimetric parameters for the main excretory organs and total body is given in Table 2 . The kidneys clearly had the

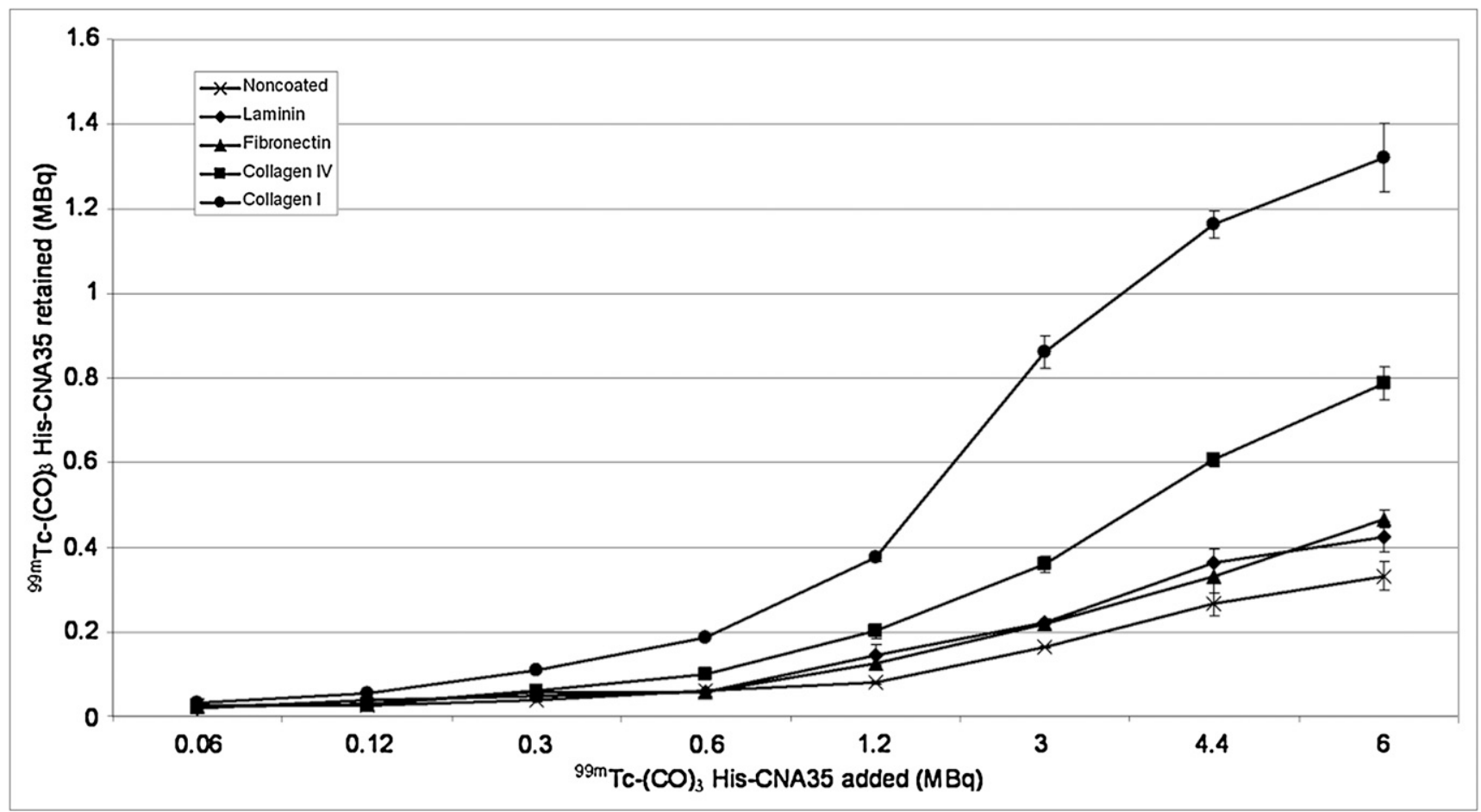

FIGURE 1. Binding of ${ }^{99 \mathrm{mTC}}-(\mathrm{CO})_{3}$ His-CNA35 to collagen I-, collagen IV-, laminin-, fibronectin-, and noncoated multiwell plates. Data are expressed as mean $\pm \operatorname{SEM}(n=3)$. 
TABLE 1

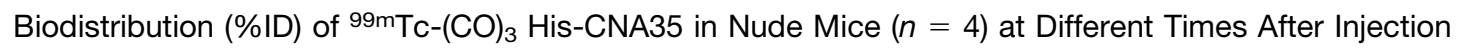

\begin{tabular}{lccrr}
\hline $\begin{array}{c}\text { Time after } \\
\text { injection (min) }\end{array}$ & Blood & Liver & \multicolumn{1}{c}{$\begin{array}{c}\text { Right } \\
\text { kidney }\end{array}$} & $\begin{array}{c}\text { Left } \\
\text { kidney }\end{array}$ \\
\hline 1 & $6.54 \pm 1.47$ & $17.30 \pm 7.01$ & $18.10 \pm 4.32$ & $8.79 \pm 0.97$ \\
10 & $3.41 \pm 0.35$ & $18.47 \pm 6.52$ & $19.73 \pm 4.20$ & $11.06 \pm 0.65$ \\
20 & $2.66 \pm 0.19$ & $17.41 \pm 5.97$ & $20.17 \pm 4.06$ & $12.71 \pm 0.64$ \\
30 & $2.33 \pm 0.23$ & $16.10 \pm 5.37$ & $20.58 \pm 4.02$ & $13.64 \pm 0.82$ \\
40 & $2.12 \pm 0.22$ & $15.47 \pm 5.30$ & $20.99 \pm 4.15$ & $14.27 \pm 0.92$ \\
50 & $1.95 \pm 0.16$ & $15.05 \pm 4.99$ & $21.00 \pm 4.12$ & $14.64 \pm 0.80$ \\
60 & $1.86 \pm 0.14$ & $14.55 \pm 4.90$ & $20.91 \pm 4.09$ & $14.87 \pm 0.90$ \\
70 & $1.79 \pm 0.15$ & $14.19 \pm 4.70$ & $21.18 \pm 4.12$ & $15.19 \pm 1.04$ \\
80 & $1.72 \pm 0.20$ & $13.89 \pm 4.74$ & $21.05 \pm 3.96$ & $15.38 \pm 0.96$ \\
90 & $1.72 \pm 0.13$ & $13.66 \pm 4.41$ & $20.73 \pm 4.04$ & $15.48 \pm 1.15$ \\
100 & $1.69 \pm 0.15$ & $13.30 \pm 4.32$ & $20.73 \pm 4.10$ & $19.85 \pm 4.20$ \\
240 & $1.73 \pm 0.34$ & $8.23 \pm 2.74$ & $17.07 \pm 3.24$ & $13.96 \pm 0.53$ \\
480 & $1.67 \pm 0.33$ & $9.34 \pm 2.31$ & $12.23 \pm 2.83$ & $15.40 \pm 2.71$ \\
1,380 & $1.49 \pm 0.25$ & $7.14 \pm 1.12$ & & $12.06 \pm 2.36$ \\
& & & & \\
\hline Data are \%ID \pm SD. & & & & \\
\hline
\end{tabular}

highest absorbed dose, followed by the liver. When calculations were done per organ, the effective dose (ED) equivalent and ED were 0.0129 and $0.00739 \mathrm{mSv} / \mathrm{MBq}$, respectively. When calculations were made per gram of tissue, the ED equivalent and ED were 0.00612 and $0.00402 \mathrm{mSv} / \mathrm{MBq}$, respectively. The total ED for a proposed single-injection dose of $500 \mathrm{MBq}$ of ${ }^{99 \mathrm{~m}} \mathrm{Tc}-(\mathrm{CO})_{3}$ His-CNA35 will be $3.70 \mathrm{mSv}$ (ED per organ) or 2.01 $\mathrm{mSv}$ (ED per gram of tissue). MIRDose 3.1 also gives estimates of the dose in other organs; for example, absorbed dose (per organ) for the lungs, ovaries, testes, and uterus (radiation-sensitive organs) are 0.00317, 0.00294, 0.00156, and $0.00289 \mathrm{mGy} / \mathrm{MBq}$, respectively.

\section{In Vivo Imaging of Tumor Vasculature in Human HT29 Colorectal Tumor Xenograft Model}

Three hours after intravenous injection of ${ }^{99 \mathrm{~m}} \mathrm{Tc}-(\mathrm{CO})_{3}$ His-CNA35, a planar static scan was obtained. Radiotracer uptake in the tumors was calculated by ROI analysis and expressed as tumor-to-background ratios. In all 10 HT29 human colorectal carcinoma xenografts, tumors were visually determinable and showed a heterogeneous uptake of
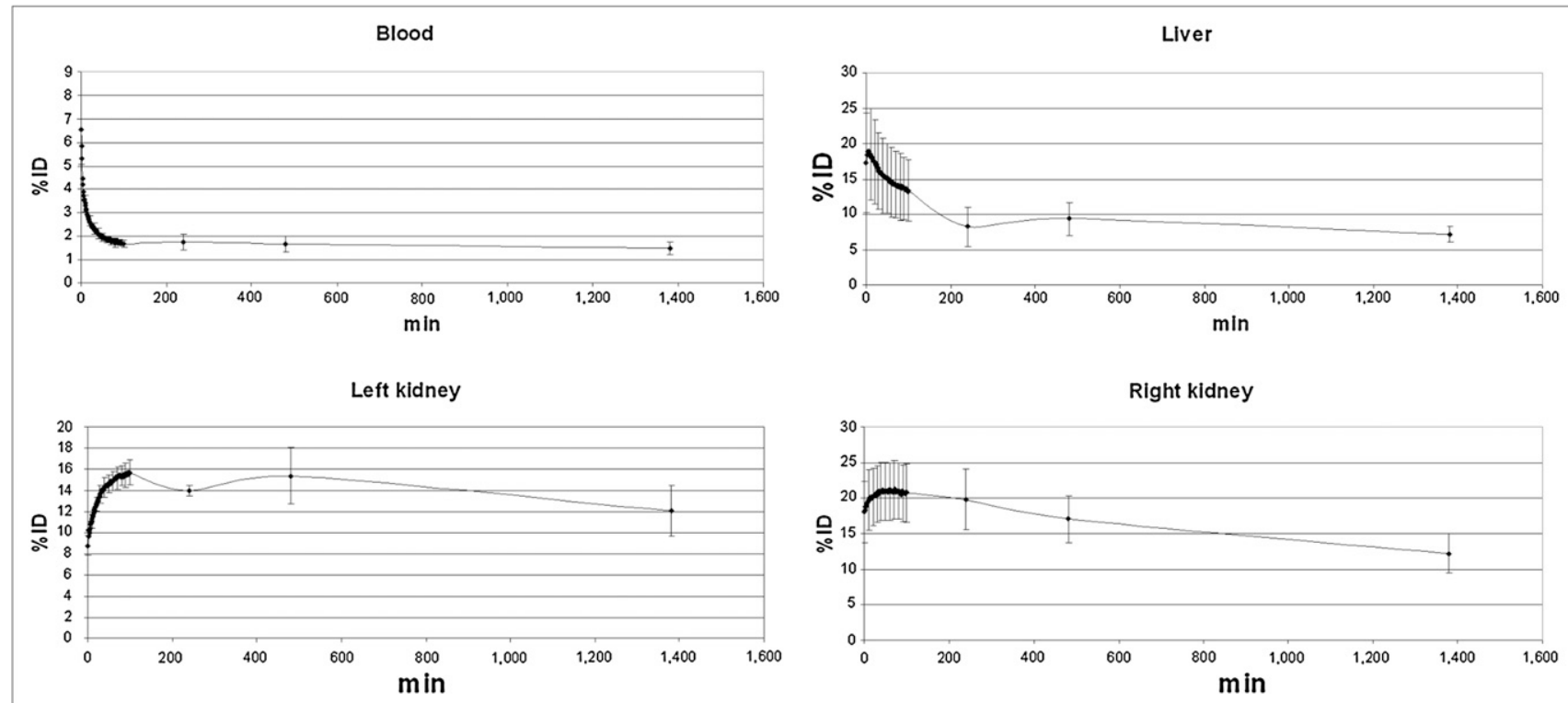

FIGURE 2. Decay-corrected ${ }^{99 m T c}-(\mathrm{CO})_{3}$ His-CNA35 time-activity curves for blood-pool, liver, right kidney, and left kidney. Planar scans were obtained from 0 to $100 \mathrm{~min}$ (dynamic study, 100 frames of $1 \mathrm{~min}$ ) and at 4, 8, and $23 \mathrm{~h}$ after injection (static study, 10 min). Kidneys show highest radiotracer uptake (right kidney more than left), followed by liver. Rapid clearance of tracer from blood was observed. 


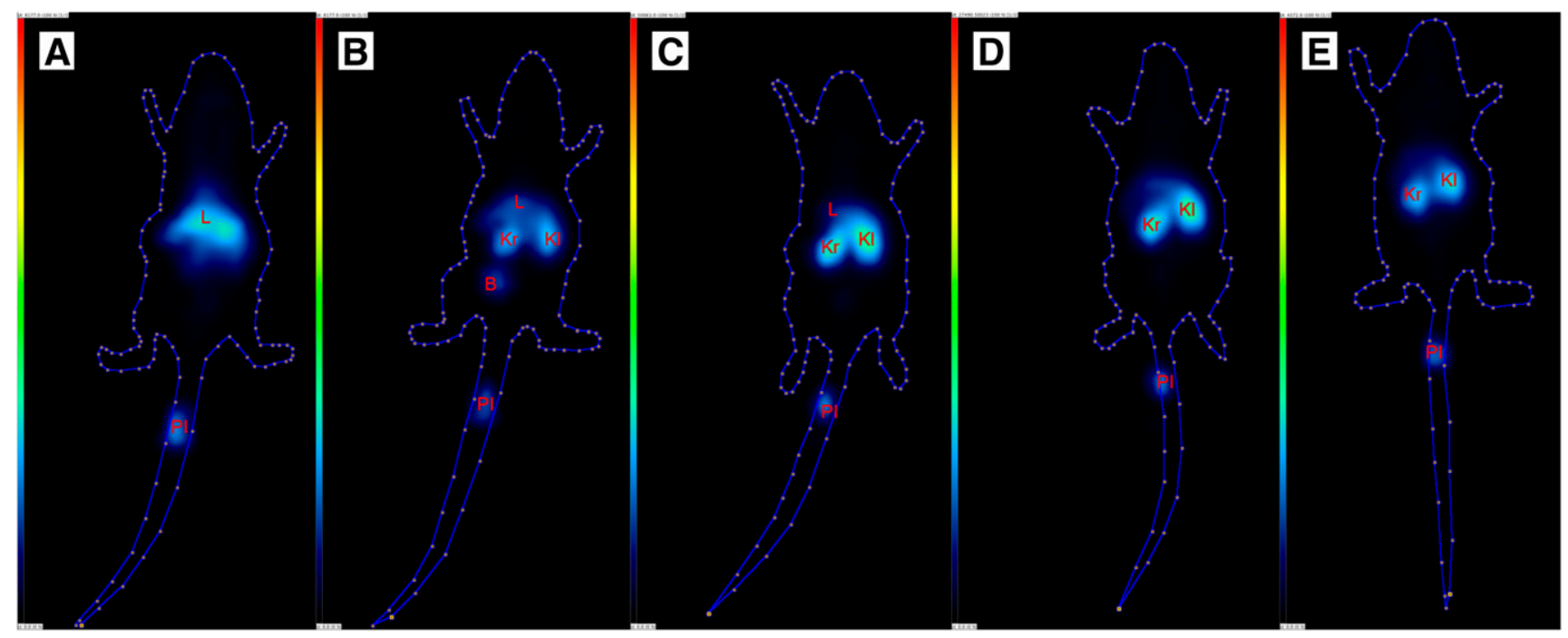

FIGURE 3. Posterior view of planar images showing in vivo biodistribution of ${ }^{99 \mathrm{mTC}}(\mathrm{CO})_{3}$ His-CNA35 in healthy mouse (37 MBq of $99 \mathrm{mTc}-$ $(\mathrm{CO})_{3}$ His-CNA35 injected), during first minute (A) and 100th minute (B) after injection of radiotracer (acquisition time, $\left.1 \mathrm{~min}\right)$ and $4 \mathrm{~h}(\mathrm{C}), 8 \mathrm{~h}$ (D), and $23 \mathrm{~h}$ (E) after injection of radiotracer (acquisition time, $10 \mathrm{~min}$ ). Liver and kidneys show highest radiotracer uptake. $\mathrm{B}=\mathrm{bladder} ; \mathrm{KI}=$ left kidney; $\mathrm{Kr}=$ right kidney; $\mathrm{L}=$ liver; $\mathrm{PI}=$ place of injection.

the radiotracer (Fig. 4). Tumor-to-background ratios varied considerably among the different animals (mean, $3.03 \pm$

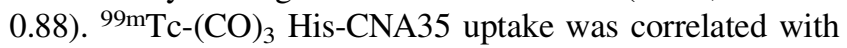
MVD $(R=0.733, P=0.016)$ and blood vessel-associated collagen IV $(R=0.758, P=0.011)$ as determined by immunohistochemistry (Fig. 5). Additionally, we found a significant inverse correlation between $\left.{ }^{99 \mathrm{~m} T c-(C O}\right)_{3}$ His-CNA35 uptake and the VMI $(R=-0.709, P=0.022)$ (Fig. 5).

\section{DISCUSSION}

In this article, we describe the successful radioactive labeling and validation of a new marker of tumor vasculature. This marker is based on binding of tumor subendothelial collagen due to the structural deficits that characterize tumor vasculature.

To our knowledge, this is the first report of the successful radioactive labeling of CNA35 to detect tumor vasculature noninvasively. Several studies have labeled CNA35 with fluorophores to detect collagen in an in vitro setting. Krahn et al. investigated the binding properties of CNA35-OG488

TABLE 2

Dosimetric Data for Intravenously Administered ${ }^{99 \mathrm{mTc}-(\mathrm{CO})_{3}}$ His-CNA35

\begin{tabular}{|c|c|c|c|c|}
\hline \multirow[b]{2}{*}{ Site } & \multicolumn{2}{|c|}{ Per organ } & \multicolumn{2}{|c|}{ Per gram of tissue } \\
\hline & $\begin{array}{c}\text { Residence } \\
\text { time (h) }\end{array}$ & $\begin{array}{c}\text { Absorbed } \\
\text { dose } \\
\text { (mGy/MBq) }\end{array}$ & $\begin{array}{c}\text { Residence } \\
\text { time (h) }\end{array}$ & $\begin{array}{c}\text { Absorbed } \\
\text { dose } \\
\text { (mGy/MBq) }\end{array}$ \\
\hline Kidneys & 2.74 & 0.138 & 1.05 & 0.0538 \\
\hline Liver & 0.91 & 0.0145 & 0.33 & 0.00593 \\
\hline $\begin{array}{l}\text { Total } \\
\text { body }\end{array}$ & 7.56 & 0.00378 & 7.56 & 0.00252 \\
\hline
\end{tabular}

and confirmed binding to collagen types I-VI, with various affinities and no binding to other extracellular matrix proteins (5). In the following in vitro study, collagen formation around human venous saphena cells was successfully demonstrated (5). Using the same molecule, Boerboom et al. successfully obtained high-resolution images of collagen in samples of human venous saphena myofibroblast cell cultures, mouse carotid artery vessels, and engineered cardiovascular constructs (6). The only in vivo study to our knowledge successfully performed fluorescent collagen labeling after intravenous injection of CNA35-OG488 (7).

In our study, labeling yields of approximately 40\%-50\% and high radiochemical purities $(\geq 95 \%)$ were obtained. The incubation of CNA35 with the ${ }^{99 \mathrm{~m}}$ Tc-tricarbonyl took place at a temperature of $37^{\circ} \mathrm{C}$ at physiologic $\mathrm{pH}$, and the radiotracer remained stable for up to $24 \mathrm{~h}$, at $4^{\circ} \mathrm{C}$ and $37^{\circ} \mathrm{C}$, in physiologic solution, cell medium, and human blood serum. Overall, preparation of ${ }^{99 \mathrm{~m}} \mathrm{Tc}-(\mathrm{CO})_{3}$ His-CNA35 was straightforward and resulted in a pure product with a good stability. The functionality and specificity of the labeled molecule were confirmed in an in vitro solid-phase binding assay in which binding of ${ }^{99 \mathrm{~m}} \mathrm{Tc}-(\mathrm{CO})_{3}$ His-CNA35 to collagen I and IV was observed, with negligible binding to fibronectin and laminin. These results confirm those obtained in a previous study (5).

The biodistribution study performed in 4 healthy nude mice revealed that the radiotracer rapidly cleared from the blood after intravenous injection and was predominantly retained in the liver and the kidneys, indicating a urinary excretory pathway. Uptake in the right kidney was higher than in the left kidney at all times. This higher uptake in the right kidney may be explained by a partial overlap of the right kidney with the liver or by the position of the right kidney in relation to the camera. The high uptake in the 


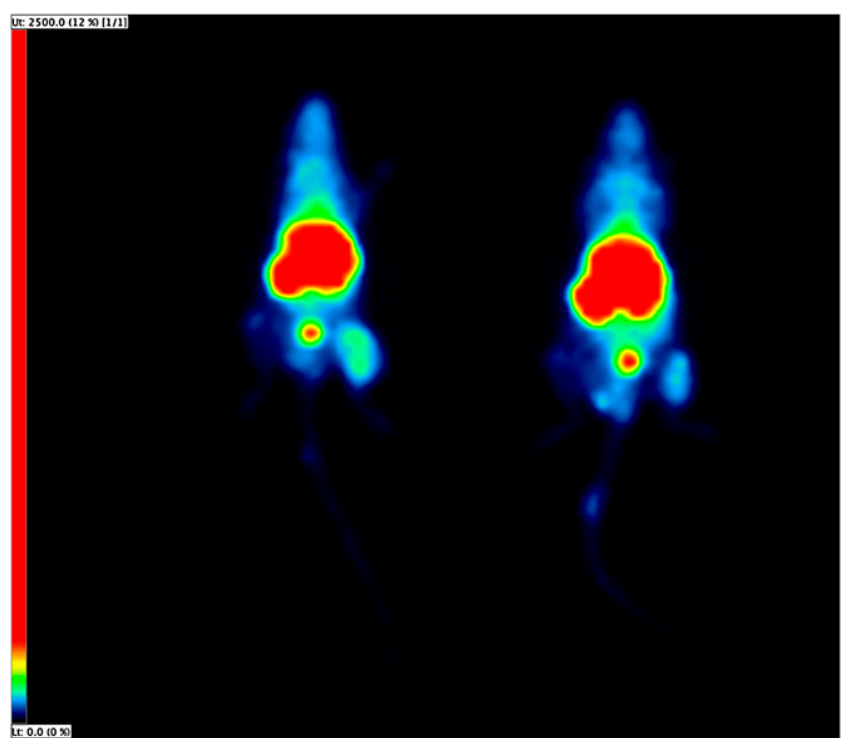

FIGURE 4. In vivo imaging of tumor vasculature in 2 HT29 human colorectal carcinoma-bearing mice (right hind leg) showing, respectively, mouse with high tumor uptake (tumor-to-background ratio, 3.94) (left) and mouse with low tumor uptake (tumor-to-background ratio, 2.63) (right).

liver and the kidneys may be due to the nature of the endothelial lining in these organs. Unlike in other organs, the blood vessels in the liver have a discontinuous endothelial coverage, and the blood vessels of the kidneys have a fenestrated endothelium $(12,13)$. As a result, labeling of collagen in the subendothelial layers of these blood vessels can occur. Megens et al. described a similar phenomenon when they studied the uptake of a fluorescently labeled variant of CNA35 in healthy and atherosclerotic arteries of mice after both ex vivo and in vivo administration (7). The administration of ${ }^{99 \mathrm{~m}} \mathrm{Tc}-(\mathrm{CO})_{3}$ His-CNA35 to patients would cause an $\mathrm{ED}$ of $0.00739 \mathrm{mSv} / \mathrm{MBq}$, corresponding to a total ED of $3.70 \mathrm{mSv}$ (ED per organ) for a patient dose of $500 \mathrm{MBq}$. These numbers are in the low range when compared with other tracers used in a clinical setting (14). However, allometric scaling from laboratory animals to humans was performed on the basis of body-organ weight. This scaling assumes that the biokinetics of compounds depend primarily on the metabolic rate of the animal, which in turn is a function of the body weight or body surface area. The EDs obtained in this study give merely an estimation of the ED that would be obtained in human patients. A detailed study in humans for a reliable estimation of the ED is still recommended.
To image tumor vasculature in vivo, human colorectal carcinoma xenografts were imaged $3 \mathrm{~h}$ after injection of

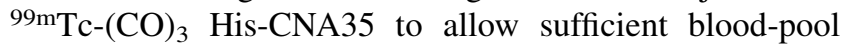
clearance. A heterogeneous uptake of the radiotracer was seen in all tumors. A significant correlation between ${ }^{99 \mathrm{~m}} \mathrm{Tc}-$ $(\mathrm{CO})_{3}$ His-CNA35 uptake and MVD was demonstrated. Further, we found a significant correlation between ${ }^{99 \mathrm{~m}} \mathrm{Tc}-(\mathrm{CO})_{3}$ His-CNA35 uptake and collagen IV that was associated with blood vessels. Additionally, we found a significant inverse correlation between ${ }^{99 \mathrm{~m}} \mathrm{Tc}-(\mathrm{CO})_{3}$ HisCNA35 uptake and the VMI. This VMI gives an estimate of the percentage of vessels that is associated with mural cells and thus an estimate of the maturity of the vessels. These results indicate that imaging using CNA35 gives a reliable estimate of existing tumor vasculature and confirm the proposed mechanism of binding in which the characteristic leakiness and immatureness of tumor blood vessels allows binding of subendothelial collagen IV and selective imaging of tumor vasculature. A limitation to our approach is the existence of capillaries with a fenestrated or discontinuous endothelium in several tissues such as kidney, endocrine gland, gastrointestinal tract, and liver $(12,13)$. Although biodistribution studies did not indicate much binding of our molecule to other organs except the kidneys and liver and all tumors were visually determinable, future studies will have to assess the extent of nonspecific binding in these organs. Discontinuous endothelia (or sinusoids) that can be found in the liver usually have an incomplete or absence of basement membrane, preventing major binding $(12,13)$.

Besides histologic and circulating markers of tumor angiogenesis, several noninvasive markers of tumor vasculature and angiogenesis have been reported that use different imaging modalities. Compounds that specifically target vascular endothelial growth factor (VEGF) and its receptors together with compounds that target integrins are the best-studied candidates to image tumor vascularization. Although several of these tracers have been tested in a preclinical and clinical setting in which they successfully visualized several types of tumors and their metastases $(15,16)$, none of these are routinely used in the clinic and few have been used to detect antiangiogenic therapy response. In 2 studies, response to antiangiogenic therapy was successfully detected using ${ }^{99 \mathrm{~m}} \mathrm{Tc}$-hydrazinonicotinamide-VEGF and ${ }^{89} \mathrm{Zr}$-ranibizumab $(17,18)$. However, because of the long biologic half-life of VEGF, labeling of VEGF or VEGF-targeting molecules with long-lived iso-

FIGURE 5. Immunohistochemical staining of CD105/Endoglin (A), collagen IV (B), and $\alpha$-SMA (C) in HT29 tumors $(\times 200)$.

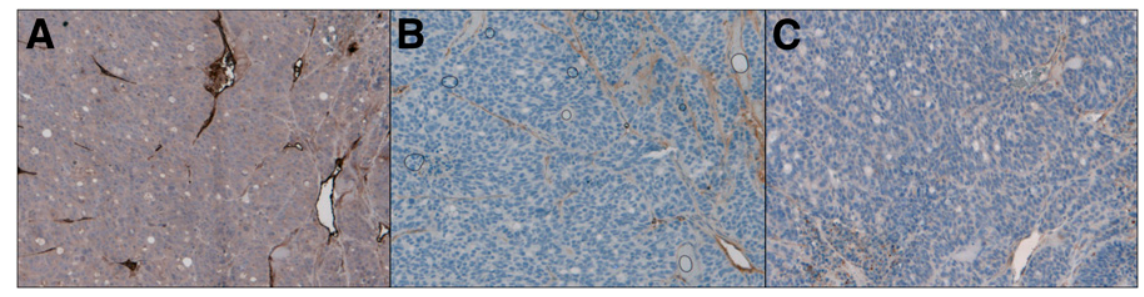


topes such as ${ }^{89} \mathrm{Zr}$ or ${ }^{111} \mathrm{In}$ is needed $(18,19)$, resulting in high radiation exposures. Using RGD (asparagine-glycineaspartic acid) peptide-based tracers, several studies also successfully evaluated treatment response to antiangiogenic therapy (20-22). However, as integrins are expressed not only on endothelial cells but on tumor cells as well $(20,23)$, and are minimally expressed on normal quiescent endothelial cells (24), RGD peptide-based tracers might not be truly representative of existing tumor vasculature. Furthermore, high uptake of ${ }^{18} \mathrm{~F}$-galacto-RGD in inflammatory lesions has been reported (25).

In future studies, we will assess the potential of ${ }^{99 \mathrm{~m}} \mathrm{Tc}-$ $(\mathrm{CO})_{3}$ His-CNA35 to evaluate the effectiveness of commonly used antiangiogenic drugs. Additionally, we will attempt to label CNA35 with PET labels such as ${ }^{68} \mathrm{Ga}$, which has the advantage of a generator-based production and a convenient half-life (68 min). Given its mechanism of action, we further propose a role for radiolabeled CNA35 in evaluating the vascular normalization window that follows antiangiogenic therapy (26). Noninvasive evaluation of the time frame of this normalization window would allow selection of the appropriate timing to administer chemotherapy.

\section{CONCLUSION}

His-tagged CNA35 was successfully labeled with ${ }^{99 m}$ Tc$(\mathrm{CO})_{3}$, resulting in a pure, functional product with a good stability. The biodistribution study demonstrated a rapid blood clearance and a predominantly renal excretion. Tumors were successfully visualized, and uptake correlated with ex vivo immunohistochemical staining of blood vessels and basement membrane and was inversely correlated with vessel maturity. Further research will determine whether ${ }^{99 \mathrm{~m}} \mathrm{Tc}-$ $(\mathrm{CO})_{3}$ His-CNA35 can provide a reliable tool for the management and planning of antiangiogenic therapy.

\section{DISCLOSURE STATEMENT}

The costs of publication of this article were defrayed in part by the payment of page charges. Therefore, and solely to indicate this fact, this article is hereby marked "advertisement" in accordance with 18 USC section 1734.

\section{ACKNOWLEDGMENTS}

CNA35-cDNA was a kind gift of Dr. Maarten Merkx, TU/e Eindhoven, The Netherlands. Part of this work was financially supported by the European Union through the grant Euregional PACT II by the Interreg IV program of Grensregio Vlaanderen-Nederland (IVAVLANED-1.20). No other potential conflict of interest relevant to this article was reported.

\section{REFERENCES}

1. Fukumura D, Jain RK. Tumor microvasculature and microenvironment: targets for anti-angiogenesis and normalization. Microvasc Res. 2007;74:72-84.

2. Homsi J, Daud AI. Spectrum of activity and mechanism of action of VEGF/ PDGF inhibitors. Cancer Control. 2007;14:285-294.

3. Ma J, Waxman DJ. Combination of antiangiogenesis with chemotherapy for more effective cancer treatment. Mol Cancer Ther. 2008;7:3670-3684.

4. Zong Y, Xu Y, Liang X, et al. A 'collagen hug' model for Staphylococcus aureus CNA binding to collagen. EMBO J. 2005;24:4224-4236.

5. Krahn KN, Bouten CVC, van Tuijl S, van Zandvoort MAMJ, Merckx M. Fluorescently labeled collagen binding proteins allow specific visualization of collagen in tissues and live cell culture. Anal Biochem. 2006;350:177-185.

6. Boerboom RA, Krahn KN, Megens RTA, van Zandvoort MAMJ, Merckx M, Bouten CVC. High resolution imaging of collagen organisation and synthesis using a versatile collagen specific probe. J Struct Biol. 2007;159:392-399.

7. Megens RT, Oude Egbrink MG, Cleutjens JP, et al. Imaging collagen in intact viable healthy and atherosclerotic arteries using fluorescently labeled CNA35 and two-photon laser scanning microscopy. Mol Imaging. 2007;6:247-260.

8. LeBleu VS, MacDonald B, Kalluri R. Structure and function of basement membranes. Exp Biol Med (Maywood). 2007;232:1121-1129.

9. Laemmli UK. Cleavage of structural proteins during the assembly of the head of bacteriophage T4. Nature. 1970;227:680-685.

10. Weidner N, Semple JP, Welch WR, Folkman J. Tumor angiogenesis and metastasis: correlation in invasive breast-carcinoma. N Engl J Med. 1991;324:1-8.

11. Spurbeck WW, Ng CY, Vanin EF, Davidoff AM. Retroviral vector-producer cellmediated in vivo gene transfer of TIMP-3 restricts angiogenesis and neuroblastoma growth in mice. Cancer Gene Ther. 2003;10:161-167.

12. Young B, Heath JW. Wheater's Functional Histology. Edinburgh, Scotland: Churchill Livingstone; 2000.

13. Aird WC. Phenotypic heterogeneity of the endothelium. Circ Res. 2007;100: $158-173$.

14. International Commission on Radiological Protection (ICRP). Radiation dose to patients from radiopharmaceuticals: addendum 3 to ICRP publication 53. ICRP publication 106. Ann ICRP. 2008;38:1-197.

15. Cai W, Chen X. Multimodality molecular imaging of tumor angiogenesis. J Nucl Med. 2008;49:113S-128S.

16. Dijkgraaf I, Boerman OC. Radionuclide imaging of tumor angiogenesis. Cancer Biother Radiopharm. 2009;24:637-647.

17. Blankenberg FG, Backer MV, Levashova Z, Patel V, Backer JM. In vivo tumor angiogenesis imaging with site-specific labeled 99mTc-HYNIC-VEGF. Eur J Nucl Med Mol Imaging. 2006;33:841-848.

18. Nagengast WB, Lub-de Hooge MN, Oosting SF, et al. VEGF-PET imaging is a noninvasive biomarker showing differential changes in the tumor during sunitinib treatment. Cancer Res. 2011;71:143-153.

19. Nagengast WB, Lub-de Hooge MN, van Straten EME, et al. VEGF-SPECT with ${ }^{11}$ In-bevacizumab in stage III/IV melanoma patients. Eur J Cancer. 2011;47: 1595-1602.

20. Jung KH, Lee KH, Paik JY, et al. Favorable biokinetic and tumor-targeting properties of ${ }^{99 \mathrm{~m}} \mathrm{Tc}$-labeled glucosamino RGD and effect of paclitaxel therapy. J Nucl Med. 2006;47:2000-2007.

21. Morrison MS, Rickets SA, Barnett J, et al. Use of a novel Arg-Gly-Asp radioligand ${ }^{18} \mathrm{~F}-\mathrm{AH} 111585$, to determine changes in tumor vascularity after antitumor therapy. J Nucl Med. 2009;50:116-122.

22. Battle MR, Goggi JL, Allen L, et al. Monitoring tumor response to antiangiogenic sunitinib therapy with ${ }^{18} \mathrm{~F}$-fluciclatide, an ${ }^{18} \mathrm{~F}$-labeled $\alpha_{\mathrm{v} \beta 3}$-integrin and $\alpha_{\mathrm{v} \beta 5}$-integrin imaging agent. J Nucl Med. 2011;52:424-430.

23. Beer AJ, Haubner R, Sarbia M, et al. Positron emission tomography using $\left[{ }^{18} \mathrm{~F}\right]$ galacto-RGD identifies the level of integrin $\alpha_{\mathrm{v} \beta 3}$ expression in man. Clin Cancer Res. 2006;12:3942-3949.

24. Brooks PC, Montgomery AM, Rosenfeld M, et al. Integrin $\alpha_{\mathrm{v}} \beta_{3}$ antagonists promote tumor regression by inducing apoptosis of angiogenic blood vessels. Cell. 1994;79:1157-1164.

25. Haubner R, Weber WA, Beer AJ, et al. Noninvasive visualization of the activated alphavbeta3 integrin in cancer patients by positron emission tomography and [ $\left.{ }^{18} \mathrm{~F}\right]$ galacto-RGD. PLoS Med. 2005;2:e70.

26. Goel S, Duda DG, Xu L, et al. Normalization of the vasculature for treatment of cancer and other diseases. Physiol Rev. 2011;91:1071-1121. 